CORRECTION

https://doi.org/10.1038/s41586-018-0070-x

\title{
Publisher Correction:
}

Programmable base editing of A๑T to $\mathrm{G} \bullet \mathrm{C}$ in genomic DNA without DNA cleavage

Nicole M. Gaudelli, Alexis C. Komor, Holly A. Rees, Michael S. Packer, Ahmed H. Badran, David I. Bryson \& David R. Liu

Correction to: Nature https://doi.org/10.1038/nature24644, published online 25 October 2017.

In this Article, owing to an error during the production process, in Fig. 1a, the dark blue and light blue wedges were incorrectly labelled as ' $\mathrm{G} \bullet \mathrm{C} \rightarrow \mathrm{T} \bullet A$ ' and ' $\mathrm{G} \bullet \mathrm{C} \rightarrow \mathrm{A} \bullet \mathrm{T}$ ', instead of ' $\mathrm{C} \bullet \mathrm{G} \rightarrow \mathrm{T} \bullet A$ ' and

' $\mathrm{C} \bullet \mathrm{G} \rightarrow \mathrm{A} \bullet \mathrm{T}$ ', respectively. Fig. 1 has been corrected online. 